\title{
ARQUIVOLOGIA E CIÊNCIA DA INFORMAÇÃO: SUBMISSÃO OU INTERLOCUÇÃO?
}

\author{
Archival Science and Information Science: submission or dialogue?
}

\begin{abstract}
Angelica Alves da Cunha Marques
Universidade de Brasília, Faculdade de Ciência da Informação, Edifício da Biblioteca Central, Entrada Leste, Mezanino, Campus Universitário Darcy Ribeiro, Brasília - DF, angelicacunha@unb.br
\end{abstract}

\begin{abstract}
Resumo
Este artigo apresenta parte das reflexões de um amplo projeto de pesquisa sobre a trajetória da Arquivologia como disciplina científica no Brasil e as suas interlocuções internacionais. Focaliza as relações da Arquivologia com outras disciplinas, especialmente no âmbito político-institucional e, particularmente, com a Ciência da Informação na contemporaneidade. Como uma pesquisa qualitativa, exploratória, descritiva e explicativa, desenvolve-se por meio de estudos bibliográfico e documental. Em leituras da Sociologia e da Filosofia, busca a compreensão histórica das classificações das ciências que subsidie a classificação da Arquivologia no contexto brasileiro atual. Os resultados demonstram relações de submissão desta disciplina à Ciência da Informação na classificação das áreas do conhecimento no Brasil, realizada pelo $\mathrm{CNPq}$, em 1984 e ainda vigente. Em uma perspectiva crítica, apresenta reflexões e questiona se essa classificação considera a história, a epistemologia, a teoria e, sobretudo, a autonomia científica da Arquivologia, em vias de consolidação nos últimos anos.
\end{abstract}

Palavras-chaves: Arquivologia; Ciência da Informação; classificação das áreas do conhecimento; campo científico; disciplina científica.

\section{"L'autonomie n'est pas un donné, mais une conquête historique, qui est toujours à recommencer.",}

(Bourdieu, 2001, p. 96)

\section{Considerações iniciais}

Pierre Bourdieu (2001) articula estruturação histórica com objetivos contemplados em projetos (habitus), lógica própria de funcionamento em um espaço (campo científico) e regulação desse espaço por meio de duas espécies de capital científico (o temporal/político e o intellectual/ puro) para a compreensão da ciência e do seu uso no contexto das lutas no mundo social e no científico. Para tanto, baseia a sua proposta na produção histórico-cognitiva do conhecimento, ao contemplar a história social da Sociologia da Ciência, as diferenças e os conflitos, além das práticas de acumulação.

Conjugando esses três conceitos, indissociáveis na perspectiva do autor, podemos retomar a trajetória histórica dos arquivos e da Arquivologia, desde o surgimen

\begin{abstract}
This paper presents a part of the reflections from a vastranging research project on the path of Archival Science as a scientific discipline in Brazil and its international dialogues. It focuses on the relations of Archival Science with other disciplines, especially in the political-institutional scope, and, particularly, with Information Science in contemporary times. As a qualitative, exploratory, descriptive, and explanatory research, it develops through bibliographic and documental studies. Reading works from Sociology and Philosophy, it seeks the historical understanding of classifications of sciences that subsidize the classification of Archival Science in the current Brazilian context. The results show relations of submission of this discipline to Information Science in the classification of knowledge areas in Brazil, caried out by the Brazilian National Council for Scientific and Technological Development (CNPq), in 1984 and still in force. In a critical perspective, it shows reflections and questions whether that classification considers Archival Science's history, epistemology, theory, and, above all, scientific autonomy, which has been going through consolidation in the last few years.
\end{abstract}

Keywords: Archival Science; Information Science; classification of knowledge areas; scientific field; scientific discipline

to daqueles na Antiguidade e a configuração desta como uma disciplina científica, a partir do século XVI (Fonseca, 2004), ou seja, um conjunto de métodos e técnicas utilizados para a compreensão (identificação), organização, preservação e recuperação dos documentos de arquivo. Auxiliar da História, da Administração eou parte da Ciência da Informação, suas relações foram da submissão à colaboração, em interlocuções não lineares e diversamente contempladas no âmbito da comunidade científica e nos diferentes contextos espaço-temporais.

Jardim (2011) reconhece a dimensão interdisciplinar como uma característica da disciplina que transita entre, pelo menos, três visões: 
- como um campo autônomo, com bases consolidadas e, de certa forma, ciência auxiliar da história;

- como uma disciplina que constitui uma subárea da ciência da informação (uma interpretação que ganha espaço especialmente no Brasil, sem maior veiculação internacional); e

- como uma disciplina científica em permanente construção, dotada de autonomia, porém exercida (ou potencialmente exercida) em diversos aspectos mediante relações interdisciplinares com a história, a administração, a ciência da informação, a bibliteconomia, a museologia, a sociologia etc. (Jardim, 2011, p. 57-58).

Esta é a perspectiva do autor, que se aproxima da nossa, conforme doravante buscaremos apresentar, mediante um breve histórico da classificação das ciências que possa nos subsidiar o entendimento das relações (de submissão e de interlocução) da Arquivologia com outras disciplinas, especialmente com a Ciência da Informação.

Este artigo é fruto de um extenso projeto de pesquisa, mais especificamente de um estágio pós-doutoral realizado no Brasil e na França (respectivamente na Universidade de Brasília e na École Nationale des Chartes, entre 2014 e 2015). Dentre os objetivos da pesquisa, nos propusemos a investigar a trajetória da Arquivologia como disciplina científica na França, considerando as suas relações interdisciplinares no âmbito político-institucional, para que pudéssemos compará-las à situação brasileira. Assim, partimos de estudos bibliográfico e documental a fím de compreender as relações entre a Arquivologia e a Ciência da Informação nos dois países, mais particularmente quanto à submissão da Arquivologia à Ciência da Informação na classificação das áreas do conhecimento no Brasil.

\section{Classificações da(s) Ciência(s)}

Diante da variedade das obras consultadas e, consequentemente das definições de "classificação" e de "classe" que encontramos, no espaço limitado de um artigo, optamos por sintetizá-las em uma definição que nos parece clara: classificação como um processo lógico (Japiassu e Marcondes, 1991; Durozoi e Roussel, 2002), do qual são definidas classes (Japiassu e Marcondes, 1991; Abbagnano, 2003) ou grupos (Santos, 1963), a partir de características comuns (Japiassu; Marcondes, 1991; Nadeau, 1999).

O pioneirismo da sistematização da classificação do conhecimento é tributado ao italiano Angelo Poliziano, humanista e poeta que, em 1941, publicou um esquema das relações entre as ciências ou áreas do conhecimento, chamado "Panepistemon" (Dahlberg, 1979, p. 353). Essa publicação teria iniciado, segundo o autor, o "“movimento' de elaboração de sistemas de classificação".

Outros estudiosos também propuseram esquemas de classificação das ciências: o italiano Mario Nizolio
(“Antibarbarus philosophicus", 1553); Francis Bacon ("De Dignitate et Augmentis Scientiarum", 1605); Ampère (1834-1843); Saint-Hilaire (1808-1861); Henry Evelelyn Bliss, dedicado aos fundamentos filosóficos sobre classificação, publicou, dentre outras obras, "The organization of Knowledge and the system of sciences" (1929), bem como um sistema de classificação bibliográfica, em 1935; e Ranghanathan, que com o seu livro "Prolegomena to library classification" (1937), apresenta regras, postulados, princípios e processos representativos de elementos de notação.

A partir de amplas definições de "classificação", alguns autores as especificam, apresentando definições para a "classificação das ciências".

Para Santos (1963, p. 274), "a unidade da ciência funda-se na unidade de seu objecto", que pode ser, segundo ele, material (concreto) ou formal (aspecto particular do objeto material, que caracteriza a ciência). Nesse sentido, "a variedade do objeto formal permitiu a especialização científica, como também a classificação das ciências" (Santos, 1963, p. 275). O autor apresenta a classificação das ciências para:

- Os antigos filósofos (a teorética ou especulativa, que contemplava a Filosofia especulativa; e a ciência prática, relacionada às atividades humanas).

- O objeto material, por sua vez, era dividido em objeto intencional (Lógica) e objeto real (Física, como ciência fundada no primeiro grau). A Matemática e a Metafísica teriam se fundado nas abstrações de segundo e terceiro grau, respectivamente. Sob essas três disciplinas, subordinavamse outras.

- Comte, que dividia a ciência em abstrata (cujo objeto era as leis) e concreta ou natural (voltada para a aplicação das leis à história efetiva).

- Spencer, para quem a Lógica e as Matemáticas eram as ciências abstratas e as ciências concretas, aquelas que davam conta dos próprios fenômenos (a Mecânica, a Física e a Química, como abstrato-concretas; a Astronomia, a Geologia, a Biologia, a Psicologia e a Sociologia, como ciências completamente concretas).

- Os autores mais modernos, em que as divergências se multiplicam, na separação e união da Ciência à Filosofia.

Diante desse breve histórico, o autor divide as ciências, segundo os seus objetos, em: ciências do objeto real (as da Natureza e as do Espírito); e ciências de objeto ideal, como as matemáticas. E, a partir desses objetos, ele apresenta uma classificação da ciência:

a) Ciências da natureza, cujo objecto é a natureza, como conjunto de todos os sêres tempo-espaciais, a realidade corporal, tanto inorgânica como orgânica, enquanto não é produto da acção humana. O corpo humano é um objecto natural.

b) Ciências do espírito (culturais), que estudam o âmbito propriamente humano da realidade, o homem em sua peculiaridade e como criador, homem do mundo da cultura, e a própria cultura. Enfim, todo o objecto cultural, que é tudo quanto êle cria ou modifica. (Santos, 1963, 276-277).

Abbagnano (2003, p. 140), por sua vez, explica que: 
Enquanto uma enciclopédia é a tentativa de dar o quadro completo de todas as disciplinas científicas e de fixar de modo definitivo as suas relações de coordenação e subordinação, uma classificação das C. tem só o intuito mais moderno de dividir as C. em dois ou mais grupos, segundo a afinidade de seus objetos ou de seus instrumentos de pesquisa.

Ao reconhecer a importância das classificações dos filósofos do Século XIX, o autor retoma alguns modelos de classificação (Abbagnano, 2003, p. 140141):

- Comte (1830): Ciências Naturais, por sua vez divididas em Ciências abstratas ou gerais (responsáveis pelas descobertas de leis que regem os diversos fenômenos) e Ciências concretas (particulares, descritivas, voltadas para a aplicação das leis à história efetiva dos seres).

- Ampère (1834): Ciências do Espírito ou noológicas e Ciências da Natureza ou cosmológicas. Amplamente aceita, essa classificação também teria sido expressa em Ciências Culturais e Ciências Naturais.

- Spencer (1864): Ciências Abstratas (Lógica formal e Matemáticas); Ciências Abstrato-concretas (Mecânica, Física e Química); e Ciências concretas (Astronomia, Mineralogia, Geologia, Biologia, Psicologia e Sociologia).

- Dilthey (1883): Ciências Naturais (dedicadas ao conhecimento causal do objeto, que permanece externo) e Ciências do Espírito (voltadas para a compreensão e revivência intrínseca do objeto, que é o homem).

- Wundt (1889): Ciências formais (Lógica e Matemática); Ciências reais (Ciências da Natureza e do Espírito).

- Windelband (1894): Ciências nomotéticas (dizem respeito à natureza e procuram descobrir leis) e Ciências idiográficas (focalizam o indivíduo historicamente).

- Rickert (1896-1902): Ciências da Natureza (caráter generalizante) e Ciências do Espírito (caráter individualizante).

- Ostwald (1908): Ciências formais, Ciências físicas e Ciências biológicas.

- Carnap (1934): Ciências formais (somente com asserções analíticas, não têm objeto) e Ciências reais ou factuais (também conteriam asserções sintéticas).

Durozoi e Roussel (2002) também retomam sinteticamente a história da classificação, sinalizando alguns posicionamentos nesse sentido:

- Dos antigos: divisões da Filosofia (Matemática e Física no âmbito da Filosofia Natural).

- Da Idade Média: São Boaventura, que propõe quatro divisões, as quatro Artes Mecânicas (Indústria e Comércio), as Belas Artes, a Filosofia (por sua vez subdividida em Gramática, Lógica e Retórica), a Filosofia Moral (Moral Individual, Moral Doméstica e Política) e a Teologia (Dogma, Moral Cristã e Mística).

- Francis Bacon: ordenação do conhecimento em torno dos campos científico, literário e teológico.

- Auguste Comte: considerando o nível de abstração das disciplinas, reconhece a Matemática, a Sociologia, a Astronomia, a Física, a Química e a Biologia. Exclui a Metafísica e a Teologia.

Ao apresentar a sua definição de ciência como "o conhecimento certo das coisas por suas causas principais. [...] um corpo de doutrina, metodicamente formado e ordenado, que constitui um ramo particular do humano saber", Brugger (1969, p. 84) também retoma a perspectiva histórica de classificação da Ciência, a partir da Escolástica, que a dividia segundo o seu objeto: material (objeto integral concreto a que se dirige) e formal (o aspecto particular sob o qual o todo é considerado). Segundo ele, a primeira divisão da Ciência, considerando o seu objeto, diz respeito à Filosofia e à Teologia, que possuem uma visão compreensiva e universal, em contraposição às "ciências particulares", por sua vez subdivididas em três grupos:

Ciências Matemáticas (Matemática);

Ciências da Natureza: foco nas coisas "por sua natureza", sem a intervenção humana;

Ciências do Espírito ou Ciências da Cultura: dedicadas às obras das atividades espirituais humanas.

Para ele, a Ciência Natural é entendida como aquela "que tem por objeto a natureza inteira, e esforça-se por conhecê-la cientificamente" (Brugger, 1969, p. 86). Em outra perspectiva, a Ciência do Espírito "ocupa-se dos vários aspectos da vida espiritual e de suas objetivações". É também conhecida como "Ciência da Cultura" "porque o homem com sua atividade criadora espiritual vai necessariamente aperfeiçoando o que é dado na natureza; seu ato criativo é já em si cultura e produz bens objetivos culturais" (Brugger, 1969, p. 87).

Pela vertente da possível aplicação do conhecimento, o mesmo autor apresenta outra classificação da Ciência: as Ciências Experimentais (no campo da experiência) e as Ciências Especulativas (voltadas para o conhecimento puro e não para a sua aplicação). No primeiro caso, ele reconhece as Ciências Exatas, quando do rigor das medições e dos cálculos matemáticos; no segundo, ele prevê as ciências puramente teoréticas, opostas àquelas normativas (práticas).

Da definição de "ciência" de Durozoi e Roussel (2002, p. 80), por sua vez, gostaríamos de sublinhar as Ciências Humanas, entendidas como "as disciplinas cujo objeto exclusivo é o homem em suas várias dimensões (história, sociologia, psicologia, etnologia etc)".

Nadeau (1999) classifica a ciência segundo três critérios:

Objeto: Ciências da Natureza, Ciências Humanas e Ciências Lógico-formais;

Método: Ciências Nomotéticas, Ciências Idiográficas, Ciência Especulativa e Ciências Experimentais;

Objetivo ou função: Ciências Fundamentais e Ciências Aplicadas.

A classificação das ciências do Século XIX teria tido as suas raízes na sistemática naturalista do século anterior (Pombo, 1998). Independentemente das classes adotadas, esta autora pontua que toda classificação das ciências possui: um agente classificativo, um mecanismo classificador, um princípio de classificação, uma multiplicidade de fins, elementos finitos, um contexto de 
classificações precedents e um produto externo da atividade classificadora.

Também as classificações das ciências são classificações reais e, enquanto tal, não escapam às irregularidades e anomalias de que só as classificações ideais estariam isentas. Quer isto dizer que, na maior parte dos casos, também elas combinam diferentes tipos de procedimentos classificatórios, umas vezes de forma essencialista, pela determinação de caracteres comuns às diferentes ciências, por exemplo, a natureza do seus objectos de estudo (Ampère) ou o fim a que se propõem (Aristóteles); outras vezes de forma estrutural, tomando por base as articulações e influências recíprocas das ciências entre si (Piaget) ou determinações internas às próprias ciências (por exemplo, as faculdades cognitivas que nelas são privilegiadamente mobilizadas (Bacon ou Diderot); outras vezes de forma genética, tomando as ciências na sua progressiva diferenciação (Comte); outras vezes ainda de forma pragmática, tendo em vista a constituição de um programa de estudos (St. Agostinho), a realização de uma enciclopédia (Chambers, Diderot) ou a organização de uma biblioteca (Leibniz). (Pombo, 1998, p. 11).

Diante da pluralidade de aspectos que podemos considerar para a classificação da(s) ciência(s), como a Arquivologia poderia ser classificada?

\section{As classificações da Arquivologia}

Se classificada conforme algumas das propostas apresentadas na seção anterior, a Arquivologia poderia ser contemplada como uma ciência prática (relacionada às atividades humanas), na visão dos antigos filósofos (Santos, 1963) ou como uma ciência do espírito (cultural), dedicada a um objeto cultural, o arquivo (Santos, 1963; Brugger, 1969). Assim, se as atuais Ciências Sociais - Humanas, também -adviessem das Ciências do Espírito (Durozoi; Roussel, 2002), os arquivos, como criação humana e como objeto de estudo da Arquivologia, seriam estudados por uma disciplina classificada nessa abordagem. ${ }^{2}$

No século XIX, a Arquivolgia poderia, porventura, se enquadrar nas Ciências Concretas, concebidas por Comte como descritivas (Abbagnano, 2003).

Ao estudarmos a sua trajetória (Marques, 2011), observamos que as relações da Arquivologia com outras disciplinas mudam ao longo do tempo, conforme os interesses que outras áreas têm pelos arquivos. $\mathrm{Na}$ Idade Média, dá-se a revitalização do papel dos arquivos, criados na Antiguidade, que passam a ser conservados para além da sua perspectiva jurídica e patrimonial (século XII). No século XIII, os arquivos passam a servir aos interesses da História e, depois do reconhecimento do valor político e administrativo dos documentos, os arquivos passam a servir aos interesses da Administração (século XIV).

Concebidos como fontes autênticas para o desenvolvimento do trabalho erudito, os documentos de arquivo são tidos como fontes de informação, inclusive sobre o passado, no século XVI. Essa perspectiva que mais uma vez aproxima os arquivos dos interesses da História é reforçada no século seguinte (XVII) com a intensificação da sua procura, em função do caráter histórico dos documentos; da elaboração de instrumentos de pesquisa e, quase que consequentemente, da organização não orgânica dos documentos (temática ou cronológica). Em continuidade a essa perspectiva histórica, há o investimento na formação paleográfica no século XVIII e a aproximação entre a Arquivologia e a Diplomática, no século XIX. Dessa maneira, são criadas escolas para atender às demandas de leitura de interpretação de documentos (vertente diplomática e paleográfica); os arquivos continuam a serviço da História; a sua função científica se fortalece; o papel do arquivista relaciona-se à disponibilização dos documentos aos usuários e à análise crítica das fontes e esse profissional é confundido com o conservador erudito e o historiógrafo.

No século XX, mais especificamente durante a Segunda Guerra Mundial, a Arquivologia passa a ser entendida como descritiva, quando entra em crise de identidade com a História, com o predomínio de uma vertente tecnicista, especialmente a partir dos anos 1930. Concebida como simultaneamente administrativa e histórica, em um primeiro momento e, diante da complexidade da produção doumental, diferentes concepções da disciplina e do seu objeto passam a coexistir, conforme a produção, organização e utilização dos arquivos nos diversos países e regiões (como, por exemplo, aqueles que a compreendem mais próxima da História, como a França; ou conjunto de técnicas para recuperar documentos, como os Estados Unidos; ou como uma (das) Ciência(s) da Informação, como em Portugal ou mesmo no Brasil - embora esta concepção não seja consensual aqui) (Marques, 2011; 2017a).

As primeiras fundações particulares para incentivo à Ciência \& Tecnologia (C\&T) e as ideias de criação de muitos conselhos de pesquisa surgem com a primeira grande guerra mundial, mediante o reconhecimento da eficácia da C\&T para a definição das batalhas (Motoyama; Nagamini, 1996). De acordo com a nossa pesquisa bibliográfica, Estados Unidos, Canadá, Reino Unido e França teriam inspirado o Brasil na institucionalização do seu Conselho Nacional de Pesquisas (Romani, 1982; Motoyama, 1985; Motoyama; Nagamini, 1996; Andrade, 1998).

Nos Estados Unidos, a primeira instituição nesse sentido é criada no século XIX e desdobrar-se-ia na $\mathrm{Na}$ tional Academy of Engineering (1964) e no Institute of Medicine (1970), hoje The National Academies of Sciences, Engineering, and Medicine (Motoyama, 1985; The National Academies of Sciences, Engineering, and Medicine, 2017a). Atualmente, essa instituição possui sete programas de pesquisa (The National Academies of Sciences, Engineering, and Medicine, 2017b), nos 
quais não identificamos qualquer ramo relacionado à Arquivologia. $^{3}$

No Canadá, o Conseil National de Recherches (CNRC) é o principal órgão de pesquisa e desenvolvimento do Governo, que, associado à indústria, promove a divulgação da pesquisa de laboratório, aplicando-a comercialmente para beneficiar a população (Conseil National de Recherches Canadá, 2017a). O CNRC possui quatro divisões integradas de programas de pesquisa e desenvolvimento (R-D) estratégicos, nas quais há 13 setores integrados, agrupados e ligados aos domínios industriais prioritários (Conseil National de Recherches Canadá, 2017b). ${ }^{4}$ Nessas divisões e setores, não verificamos interesses arquivísticos.

No Reino Unido, o Research Councils UK (RCUK) integra os conselhos de pesquisa, que buscam benefícios para suas comunidades acadêmicas e para o Governo, principalmente (Research Councils UK, 2017a). São sete conselhos de pesquisa responsáveis por investir o dinheiro público na pesquisa, para o avanço do conhecimento e a geração de novas ideias. ${ }^{5}$ Desses, há o conselho de Artes e Humanidades, que abrange, entre outras áreas, Estudos de Biblioteconomia, de Informação e de Museus (Research Councils UK, 2017b).

Na França, a primeira instituição para equipar laboratórios é criada em 1901, complementada, mais tarde (1922) pelo Office Nationale des Recherches Scientifiques et des Inventions (ONRSI); pela Caisse Nationale des Sciences (CNS), em 1930; pelo Conseil Supérieur de la Recherche Scientifique (CSRS), em 1933; pelo Centre de la Recherche Scientifique Appliqué (CNRSA), em 1938; e por um serviço central em torno do Centre National de la Recherche Scientifique (CNRS), em 1939 (Motoyama; Nagamini, 1996). O CNRS caracteriza-se, atualmente, como um organismo público de pesquisa, subordinado ao Ministère de l'Éducation nationale, de l'Enseignement supérieur et de la Recherche. É composto por dez institutos, dentre os quais o Instituto de Ciências Humanas e Sociais e o Instituto de Ciências da Informação e suas interações. $\mathrm{O}$ primeiro abriga interesses históricos, sociais, antropológicos, filosóficos e políticos (não há especificação para a Arquivologia, que é entendida na perspectiva histórica); o segundo, contempla a Ciência da Informação próxima à Informática (Centre national de la recherche scientifique, 2017). ${ }^{6}$

No Brasil, a história do Conselho Nacional de Desenvolvimento Científico e Tecnológico (CNPq) começa nos anos 1920, com a Academia Brasileira de Ciências $(\mathrm{ABC})$, que inaugura a ideia da necessidade de criação de um conselho de pesquisas (Conselho Nacional de Pesquisa e Desenvolvimento Científico e Tecnológico, 1984; Motoyama, 1985; Motoyama; Nagamini, 1996), que seria criado em 1951.
Já no contexto da sua criação, o CNPq estrutura a base do instrumento para a classificação das áreas do conhecimento, tendo em vista o fomento à pesquisa. Assim, a Tabela de Áreas do Conhecimento (TAC) é vislumbrada, concebida e passa por atualizações diante de demandas relacionadas à incorporação de áreas inicialmente não contempladas. Depois de reconhecer alguns setores como áreas do conhecimento a partir de 1951, o CNPq publica três tabelas (1976, 1978 e 1984), que passam por revisões nos anos 1990 e na primeira década do século XXI.

Na primeira TAC, a Ciência da Informação é classificada como uma subárea da Comunicação e a Arquivologia não aparece. Na segunda TAC, "Ciência da Informação, Biblioteconomia e Arquivologia" denominam uma área, dentro da qual "Organização de Arquivos" é especialidade da subárea "Tratamento da Informação". Na terceira TAC, a Arquivologia é concebida como subárea da área Ciência da Informação, por sua vez inserida na Grande Área "Ciências Sociais Aplicadas". Em uma proposta de revisão da última TAC, em 2005, Ciência da Informação e Arquivologia são propostas como duas áreas distintas, inseridas na Grande Área "Ciências Socialmente Aplicáveis". A Arquivologia teria quatro subáreas: Fundamentos de Arquivologia; Gestão Arquivística; Métodos Arquivísticos; e Arquivologia Especializada. Contudo, esta proposta de revisão não se concretizou (Conselho Nacional de Desenvolvimento Científico e Tecnológico, 1978; 1984; Romani, 1982); Souza, 2004; 2005; 2008; Souza e Stumpf, 2009; Marques, 2016).

Portanto, o que temos hoje, no Brasil, é uma classificação das áreas do conhecimento (em sua terceira versão), que reconhece a Ciência da Informação como uma área, na qual a Arquivologia é tida como uma das suas subáreas (Conselho Nacional de Pesquisa e Desenvolvimento Científico eTecnológico, 1984).

Esta classificação político-institucional para fins de fomento à pesquisa não é consensualmente aceita pela comunidade científica. Marques (2013) afirma que estudiosos da Ciência da Informação e da Arquivologia reconhecem (ou não) as suas relações em, pelo menos, três perspectivas: 1) autores que ignoram a trajetória histórica dos arquivos e da Arquivologia e nem a consideram cientificamente, como Le Coadic (1996, p. 14); 2) autores que concebem a Arquivologia como uma parte da Ciência da Informação (Pinheiro, 1999; Silva et al, 1999); 3) e autores que demarcam a autonomia da Arquivologia e reconhecem, em níveis e graus diversos, as suas relações com a Ciência da Informação (Jardim; Fonseca, 1995; Silva, 1996; Marques, 2007; Araújo, 2010; Domínguez, 2017).

\section{Inquietações e questionamentos}

As TACs se aproximariam do que Abbagnano (2003) denomina de "enciclopédia" ou seria uma classificação 
no sentido mais geral, dividindo as disciplinas e as agrupando conforme a afinidade dos seus objetos?

Marques (2017a) nos convida a pensar se "a classificação da Ciência da Informação e da Arquivologia nas tabelas de áreas do conhecimento (TACs), pelo CNPq, reflete as trajetórias históricas e as configurações atuais dessas disciplinas no Brasil". Em sua análise, a autora afirma que as TACs no Brasil parecem se aproximar mais da perspectiva enciclopédica, com a fixação das relações entre as disciplinas, do que da divisão científica em grupos, conforme seria pela afinidade dos objetos e dos instrumentos de pesquisa das áreas do conhecimento, respeitando as singularidades das diversas disciplinas científicas.

A classificação das áreas do conhecimento no Brasil tem, como agentes classificativos, pesquisadores representantes das diversas áreas, que utilizam a hierarquização dessas áreas como mecanismo classificador, segundo princípios de classificação que não nos parecem claros - como, por exemplo, a ordem histórica da constituição e diferenciação das disciplinas; ou a natureza dos objetos estudados, como propõe Pombo (1998) -, tendo fins práticos, ao conjugar disciplinas constituídas e disciplinas em vias de constituição, num contexto de classificações precedentes, do próprio $\mathrm{CNPq}$, gerando esquemas, conhecidos como TACs. (Marques, 2017a, p. 112).

Afinal, qual é a especialização científica da Ciência da Informação não verificada na Arquivologia que justifica a atual classificação desta como subárea daquela? Mais especificamente, qual é o objeto formal da Ciência da Informação que justifica a sua autonomia científica em relação às outras disciplinas, tal como nos propõem Santos (1963) e Brugger (1969)?

Definido de forma ampla, o objeto da Ciência da Informação transita entre a informação e o seu tratamento, o seu fluxo, a sua recuperação e o seu acesso. Definido em uma perspectiva mais específica, o objeto da Arquivologia é representado pelo arquivo ou pelo documento de arquivo (Schmidt, 2012). Assim, não verificamos especialização na Ciência da Informação em relação à Arquivologia que paute a sua concepção como uma área que a abrigue como uma subárea, tal como aparece na TAC em vigor (Conselho Nacional de Desenvolvimento Científico e Tecnológico, 1984).

Semelhantemente, observamos princípios científicos na Arquivologia, não constatados na literatura da Ciência da Informação (Kuroki, 2016; Ramírez Aceves, 2017), além de uma longevidade histórica dos arquivos e da Arquivologia, também não verificada na recente história da Ciência da Informação como disciplina (Marques, 2011). Nesse sentido, o questionamento de Marques (2016, p. 180) parece pertinente: "Ainda manter-se-ia a Arquivologia, uma disciplina científica que vem se formando desde o século XVI (Fonseca, 2004), subordinada à Ciência da Informação, uma disciplina nascida na segunda metade do século XX?" Ou seja, "Esta questão cronológica legitimaria a emancipação da Arquivologia como uma área do conhecimento independente da Ciência da Informação ou não seria suficiente para tal a despeito do papel coadjuvante da Arquivologia no Campo da Informação, como nos sugere Schmidt (2012)?”

Contudo, a Ciência da Informação ainda tem maior visibilidade político-institucional que a Arquivologia, possivelmente em razão da propagação do discurso informacional nos arquivos mediante os aspectos apontados por Schmidt (2012, p. 260):

[...] institucionalização da Ciência da Informação e sua "capacidade" científica "não alcançada" pela Arquivologia, a "emergência" da Sociedade da Informação e a necessidade em renovar o discurso teórico que aqui se configurava [em meados da década de 1990], baseado majoritariamente por ideias europeias advindas de países como França e Espanha.

\section{Inconclusiva classificação}

Ao final da segunda seção deste artigo, questionamos como a Arquivologia poderia ser classificada, considerando a pluralidade de aspectos que perpassam a classificação da(s) ciência(s). Para tal, imprescindível se faz a compreensão da Arquivologia como uma disciplina científica, nem sempre assim contemplada por todos, diante dos interesses que outras áreas têm pelos arquivos. Assim, a subordinação ou a autonomia da Arquivologia quanto às outras disciplinas parece variar de acordo com esses interesses.

A partir das recomendações de Bourdieu (2001), observamos que imprescindível se faz para a configuração de um campo científico a conjugação dos seus habitus e dos seus capitais, para que espaço, tradição e regulação definam uma lógica própria de funcionamento ao longo do tempo, mediante a dinâmica de uma comunidade. Esta dinâmica parece adequada para todas as disciplinas e é assim que, inspirados nesse autor e pautados na trajetória da Arquivologia apresentada em sua literatura (Fonseca, 2005; Silva et al, 1999, Marques, 2011), concordamos com Marques (2016) acerca da autonomia dessa disciplina, inclusive em relação à Ciência da Informação, por mais que esta tenha alcançado visibilidade na contemporaneidade.

Considerando a trajetória histórica e epistemológica da Arquivologia, entendemos que esta disciplina não pode ser compreendida como uma parte da Ciência da Informação, ainda que esta última tenha conquistado importantes espaços acadêmico-institucionais nos últimos anos, de maneira a abrigar cursos e pesquisas arquivísticos em universidades e programas de pós-graduação. Nesse sentido, pensamos que a manutenção da subordinação da Arquivologia à Ciência da Informação camufla conquistas seculares daquela disciplina, encobrindo suas peculiaridades teóricas e epistemológicas por trás de questões políticoinstitucionais. (Marques, 2016, p. 181).

Assim, a propagação do discurso informacional nos arquivos lembrada por Schmidt (2012), aliada à baixa 
visibilidade político-institucional da Arquivologia não representa os avanços histórico-epistemológicos e teóricos da disciplina, dificultando a sua ressonância (Marques, 2017b).

Diante dessas incongruências, esperamos que a classificação das áreas do conhecimento no Brasil seja inconclusiva e brevemente substituída, não somente pelas limitações típicas de qualquer esquema classificatório, como também por não refletir os avanços de uma disciplina que tem história e teoria e que anseia por interlocução, recusando a subordinação.

\section{Notas}

(1) "A autonomia não é um dado, mas uma conquista histórica que está sempre a recomeçar" (Bourdieu, 2001, p. 96, tradução nossa).

(2) Mas esta relação entre as Ciências do Espírito e as Ciências Sociais não é linear, tampouco consensual, como bem demonstra Jaeschke (2006). Esse estudioso lembra que no século XVIII ainda não havia a dualidade entre as Ciências Naturais e as Ciências do Espírito, até porque muito do que hoje conhecemos como "Ciência Humana" não era considerado ciência, mas arte. Com o Positivismo, a referida dualidade é construída no final do século XIX, dando às Ciências Naturais um caráter explanatório na revelação das leis subjacentes e na identificação do universal, e às Ciências do Espírito, uma perspectiva "compreensiva" na apreensão do individual no seu caráter peculiar. "Ocorre que a diferença entre 'explicar' e 'compreender' não é tão fecunda como provavelmente seria desejável; além disso, ela não pode ser mantida com precisão no plano terminológico nem no idioma alemão, ao menos não enquanto a compreensão não for introduzida expressamente como compreensão de 'sentido'. $\mathrm{Se}$, contudo, quisermos servir-nos dessa palavra que adquiriu foros de cidadania também no século XIX, uma outra descoberta também se revela inevitável: o 'sentido' sempre é algo espiritual" (Jaeschke, 2006, p. 124).

(3) Behavioral and Social Sciences and Education, Earth and Life Studies, Engineering and Physical Sciences, Health and Medicine, Policy and Global Affairs, Transportation Research Board, and the Gulf Research Program (The National Academies of Sciences, Engineering, and Medicine, 2017b).

(4) Transports et de la fabrication (Centre de recherche en aérospatiale et Centre de recherche sur l'automobile et les transports de surface); Génie (Centre de recherche sur l'énergie, les mines et l'environnement; et Centre de recherche en con/OCRE Centre de recherche en génie océanique, côtier et fluvial); Sciences de la vie (Centre de recherche en développement des cultures et des ressources aquatiques; Centre de recherche en thérapeutique en santé humaine; Centre de recherche sur les dispositifs médicaux); Technologies émergentes (Centre de recherche en électronique et photonique avancées; Centre de recherche en technologies numériques; Science des mesures et étalons; Centre de recherches en nanotechnologie; CNRC Herzberg, Astronomie et astrophysique; Technologies de sécurité et de rupture) (Conseil National de Recherches Canadá, 2017b).
(5) Arts \& Humanities Research Council; Biotechnology \& Biological Sciences Research Council; Engineering \& Physical Sciences Research Council; Economic \& Social Research Council; Medical Research Council; Natural Environment Research Council; Science and Technology Facilities Council (Research Councils UK, 2017b).

(6) Institut des sciences biologiques (INSB); Institut de chimie (INC); Institut écologie et environnement (INEE); Institut des sciences humaines et sociales (INSHS); Institut des sciences de l'information et de leurs interactions (INS2I); Institut des sciences de l'ingénierie et des systèmes (INSIS); Institut national des sciences mathématiques et de leurs interactions (INSMI); Institut de physique (INP); Institut national de physique nucléaire et physique des particules (IN2P3); Institut national des sciences de l'univers (INSU) (Centre national de la recherche scientifique, 2017).

\section{Referências}

Abbagnano, Nicola (2003). Dicionário de Filosofia. 4 ed. São Paulo: Martins Fontes, 2003.

Andrade, Ana Maria Ribeiro de (1998). História e fonte para a história do CNPq. // Museu de Astronomia e Artes Afins (MAST/CNPq). Arquivo CNPq (Acervo MAST): inventário sumário. Rio de Janeiro: MAST, 1998.

Araújo, Carlos Alberto Ávila (2010). Ciência da Informação como campo integrador para as áreas de Biblioteconomia, Arquivologia e Museologia. // Informação \& Informação 15:1 (2010) 173-189.

Bourdieu, Pierre (2001). Science de la science et réflexivité: Cours du Collège de France 2000-2001. Paris: Raisons d'agir, 2001.

Brugger, Walter (1969). Dicionário de Filosofia. 2 ed. São Paulo: Herder, 1969.

Centre national de la recherche scientifique (2017). Présentation (2017). http://www.cnrs.fr/fr/organisme/presentation.htm (1610-2017).

Conseil National de Recherches Canadá (2017a). À propos du CNRC. (2017a). http://www.nrccnrc.gc.ca/fra/apropos/index.html (16-10-2017).

Conseil National de Recherches Canadá (2017b). Survol. (2017b) http://www.nrc-cnrc.gc.ca/fra/apropos/vue_ensemble/index.html (16-10-2017).

Conselho Nacional de Desenvolvimento Científico e Tecnológico (1978). Classificação das áreas do conhecimento. // Cadernos de Informação em Ciência e Tecnologia 1 (1978).

Conselho Nacional de Desenvolvimento Científico e Tecnológico (1984). Áreas do conhecimento: classificação. Brasília: CNPq, 1984.

Cruz Domínguez, Silvana Elisa (2017). La permanente defense de la Archivística por su autonomía. // Rendón Rojas, Miguel Ángel. (Org.). La Archivística y la ciencia de la información documental: autonomía e interdependencias. México: UNAM, Instituto de Investigaciones Bibliotecológicas y de la Información, 2017, 3958 .

Dahlberg, Ingetraut (1979). Teoria da classificação, ontem e hoje. // Conferência Brasileira de Classificação Bibliográfica. Anais. Rio de Janeiro: IBICT (1979) 352-370.

Durozoi, Gérard; Roussel, André (2002). Dicionário de Filosofia. 4 ed. Campinas: Papirus, 2002.

Fonseca, Maria Odila Kahl (2004). Arquivologia e Ciência da Informação: (re)definição de marcos interdisciplinares. Univer- 
sidade Federal do Rio de Janeiro, Rio de Janeiro, 2004. Tese de doutorado em Ciência da Informação.

Jaeschke, Walter (2006). As Ciências Naturais e as Ciências do Espírito na era da globalização. // Veritas 51:1 (2006) 121-132.

Japiassu, Hilton; Marcondes, Danilo (1991). Dicionário básico de Filosofia. 2 ed. Rio de Janeiro: Zahar, 1991

Jardim, José Maria (2011). A pesquisa como fator institucionalizante da Arquivologia enquanto campo científico no Brasil. // Marques, Angelica Alves da Cunha; Roncaglio, Cynthia; Rodrigues, Georgete Medleg. A formação e a pesquisa em Arquivologia nas universidades públicas brasileiras: I Reunião Brasileira de Ensino e Pesquisa em Arquivologia. Brasília: Thesaurus, (2011) 53-75.

Jardim, José Maria; Fonseca, Maria Odila (1995). As relações entre a Arquivística e a Ciência da Informação. // INFORMARE 1:1 (1995) 41-50.

Kuroki, Ívina Flores Melo (2016). Demarcações conceituais dos princípios científicos da Arquivologia e da Ciência da Informação: contribuições para a configuração científica das disciplinas no Campo da Informação. Universidade de Brasília, Brasília, 2016. Dissertação de mestrado em Ciência Informação.

Le Coadic, Yves-François (1996). A Ciência da Informação. Brasília: Briquet de Lemos, 1996.

Marques, Angelica Alves da Cunha (2011). Interlocuções entre a Arquivologia nacional e a internacional no delineamento da disciplina no Brasil. Universidade de Brasília, Brasília, 2011. Tese de doutorado em Ciência da Informação.

Marques, Angelica Alves da Cunha (2013). Os lugares da arquivologia no campo da informação. // Ciência da Informação (Online) 42 (2013) 109-123.

Marques, Angelica Alves da Cunha (2016). Arquivologia e Ciência da Informação: de mãos dadas? // Informação \& Sociedade (UFPB. Online) 26 (2016) 169-184.

Marques, Angelica Alves da Cunha (2017a). Ciência da Informação e Arquivologia: áreas do conhecimento? // Rendón Rojas, Miguel Ángel. (Org.). La Archivística y la ciencia de la información documental: autonomía e interdependencias. México: UNAM, Instituto de Investigaciones Bibliotecológicas y de la Información (2017a) 99-117.

Marques, Angelica Alves da Cunha (2017b). A investigação científica em Arquivologia e a sua busca de identidade. // Pesquisa Brasileira em Ciência da Informação e Biblioteconomia 12:2 (2017b) 77-89.

Motoyama, Shozo (1985). A gênese do CNPq. // Revista da Sociedade Brasileira de História da Ciência 2 (1985) 27-46.

Motoyama, Shozo; Nagamini, Marilda (1996). CNPq e CNRS: duas histórias numa perspectiva comparada (1996). // Hamburger, Amélia Império. (et al). A ciência nas relações Brasil-França (1850-1950). São Paulo: Editora da Universidade de São Paulo; FAPESP (1996) 331-359.

Nadeau, Robert (1999). Vocabulaire technique et analytique de l'epistemologie. Paris: Puf, 1999.

Pinheiro, Lena Vânia Ribeiro (1999). Campo interdisciplinar da Ciência da Informação: fronteiras remotas e recentes. // Pinheiro, Lena Vânia Ribeiro (Org.). Ciência da Informação, Ciências Sociais e Interdisciplinaridade. Brasília/Rio de Janeiro: IBICT/DDI/DEP (1999) p. 155-182.

Pombo, Olga (1998). Da classificação dos seres à classificação dos saberes. // Revista da Biblioteca Nacional de Lisboa 2 (1998) 1933

Ramírez Aceves, Merizanda M. C. (2017). Apuntes sobre los fundamentos científicos de la Archivística. // Rendón Rojas, Miguel
Angel. (Org.). La Archivística y la ciencia de la información documental: autonomía e interdependencias. México: UNAM, Instituto de Investigaciones Bibliotecológicas y de la Información (2017) 59-98.

Research Councils UK (2017a). RCUK Aims \& Organisation. (2017a). http://www.rcuk.ac.uk/about/aboutrcuk/aims/ (16-102017).

Research Councils UK (2017b). Areas of Research. (2017b). http://www.rcuk.ac.uk/research/areas/ 16-10-2017).

Romani, Jacqueline Pitangui (1982). O Conselho Nacional de Pesquisas e Institucionalização da Pesquisa Científica no Brasil. // Schwartzman, Simon (Org.). Universidades e instituições científicas no Rio de Janeiro. Brasília: CNPq, 1982, 137-167.

Santos, Mário Ferreira dos (1963). Dicionário de Filosofia e Ciências Culturais. São Paulo: Matese, 1963.

Schmidt, Clarissa Moreira dos Santos (2012). Arquivologia e a construção do seu objeto científico: concepções, trajetórias, contextualizações. Universidade de São Paulo, São Paulo, 2012. Tese de doutorado em Ciência da Informação.

Silva, Armando Malheiro da. et al (1999). Arquivística: teoria e prática de uma Ciência da Informação. Porto: Afrontamento, 1999.

Silva, Junia Guimarães e (1996). Socialização da informação arquivística: a viabilidade do enfoque participativo na transferência da informação. Universidade Federal do Rio de Janeiro, Rio de Janeiro, 1996. Dissertação de mestrado em Ciência da Informação.

Souza, Rosali Fernandez de (2004). Áreas do conhecimento. // DataGramaZero: Revista de Ciência da Informação 5:2 (2004).

Souza, Rosali Fernandez de (2005). Tabelas de áreas do conhecimento: proposta de metodologia do trabalho de revisão. [Rio de Janeiro]: 2005. (Fundo CNPq)

Souza, Rosali Fernandez de (2006). Organização e representação de áreas do conhecimento em Ciência e Tecnologia: princípios de agregação em grandes áreas segundo diferentes contextos de produção e uso da informação. // Encontros Bibli: Revista Eletrônica de Biblioteconomia e Ciência da Informação n. esp., (2006).

Souza, Rosali Fernandez de (2008). A Ciência da Informação como área do conhecimento e de fomento no CNPq. // Anais do Encontro Nacional de Ensino e Pesquisa em Informação. 8 (2008).

Souza, Rosali Fernandez de; Stumpf, Ida Regina Chitto (2009). Ciência da Informação como área do conhecimento: abordagem no contexto da pesquisa e da Pós-graduação no Brasil. // Perspectivas em Ciência da Informação 14: n. especial (2009) 41 58.

The National Academies of Sciences, Engineering, and Medicine (2017a). History of the National Academies. (2017a). http://www.nationalacademies.org/about/history/index.html (1610-2017).

The National Academies of Sciences, Engineering, and Medicine (2017b). History of the National Academies. (2017b). What We Do. Estados Unidos, 2017b http://www.nationalacademies.org/about/whatwedo/index.html (16-10-2017) 
Copyright: (C) 2017 Marques. This is an open-access article distributed under the terms of the Creative Commons CC Attribution-ShareAlike (CC BY-SA), which permits use, distribution, and reproduction in any medium, under the identical terms, and provided the original author and source are credited. 